\title{
Matricaria recutita Gel
}

National Cancer Institute

\section{Source}

National Cancer Institute. Matricaria recutita Gel. NCI Thesaurus. Code C118448.

A gel-based formulation containing an extract of the herb Matricaria chamomilla (M. recutita or German chamomile), which is native to eastern and southern Europe, belongs to the Asteraceae family and is high in flavonoids, with potential anti-inflammatory, skin protective, moisturizing, anti-bacterial, anti-oxidant and calming activities. M. recutita extract contains flavonoids, including apigenin, luteolin and quercetin, as well as coumarins, herniarin, umbelliferone, anthemic acid, anthemidine, tannin and matricarin. Upon topical application of the Matricaria recutita gel, the active ingredients in the chamomile may exert anti-inflammatory, calming and anti-oxidant effects on the skin and may protect the skin against radiotherapy-induced dermatitis. 